OPEN ACCESS

Edited by:

Hector Mora Montes,

University of Guanajuato, Mexico

Reviewed by:

Mira Edgerton,

University at Buffalo, United States

Monika Staniszewska,

Warsaw University of Technology,

Poland

${ }^{*}$ Correspondence:

Zuobin Zhu

geneticszzb@163.com

Hongchun $\mathrm{Li}$

13775891123@163.com

tThese authors have contributed equally to this work and share first

authorship

Specialty section:

This article was submitted to Fungi and Their Interactions,

a section of the journal

Frontiers in Microbiology

Received: 09 October 2019 Accepted: 22 November 2019 Published: 03 December 2019

Citation:

Li Y, Shan M, Yan M, Yao H, Wang Y, Gu B, Zhu Z and Li H (2019) Anticandidal Activity

of Kalopanaxsaponin A: Effect on Proliferation, Cell Morphology, and Key Virulence Attributes

of Candida albicans.

Front. Microbiol. 10:2844. doi: 10.3389/fmicb.2019.02844

\section{Anticandidal Activity of Kalopanaxsaponin A: Effect on Proliferation, Cell Morphology, and Key Virulence Attributes of Candida albicans}

\author{
Ying Lit', Mingzhu Shan ${ }^{1+}$, Mingju Yan1, Huankai Yao ${ }^{2}$, Yuechen Wang ${ }^{3}$, Bing Gu ${ }^{1,4}$, \\ Zuobin $\mathrm{Zhu}^{3 *}$ and Hongchun $\mathrm{Li}^{1,4 *}$
}

${ }^{1}$ School of Medical Technology, Xuzhou Medical University, Xuzhou, China, ${ }^{2}$ Jiangsu Key Laboratory of New Drug Research and Clinical Pharmacy, School of Clinical Pharmacy, Xuzhou Medical University, Xuzhou, China, ${ }^{3}$ Department of Genetics, Xuzhou Medical University, Xuzhou, China, ${ }^{4}$ Department of Laboratory Medicine, Affiliated Hospital of Xuzhou Medical University, Xuzhou, China

Background: The pathogenicity of Candida albicans is attributed to various virulence factors including adhesion to the surface of epithelial cells or mucosa, germ tube formation, hyphal morphogenesis, development of drug resistant biofilms, and so on. The objective of this study was to investigate the effects of Kalopanaxsaponin A (KPA) on the virulence of $C$. albicans.

Methods: The effect of KPA on the virulence of $C$. albicans was characterized by an XTT reduction assay and fluorescent microscopic observation. The action mechanism was further explored using GC/MS system and BioTek Synergy2 spectrofluorophotometry. The cytotoxicity and therapeutic effect of KPA were evaluated by the Caenorhabditis elegans-C. albicans infection model in vivo.

Results: The minimum inhibitory concentration (MIC) of KPA was $8 \sim 16 \mu \mathrm{g} / \mathrm{mL}$ for various genotypes of $C$. albicans. The compound was identified as having remarkable effect on the adhesion, morphological transition and biofilm formation of $C$. albicans. The results of fluorescent microscopy and GC/MS system suggested that KPA could promote the secretion of farnesol by regulating the expression of Dpp3 and decrease the intracellular CAMP level, which together inhibited morphological transition and biofilm formation. Notably, KPA showed low toxicity in vivo and a low possibility of developing resistance.

Conclusion: Our results demonstrated that KPA had remarkable efficacy against C. albicans pathogenicity, suggesting that it could be a potential option for the clinical treatment of candidiasis.

\section{Keywords: Kalopanaxsaponin A, Candida albicans, virulence factors, hyphae, farnesol, cAMP}

Abbreviations: AMB, amphotericin B; cAMP, cyclic adenosine monophosphate; DMSO, dimethyl sulfoxide; GC-MS, gas chromatography-mass spectrometry; KPA, Kalopanaxsaponin A; MIC, minimal inhibitory concentration; SD, standard deviations. 


\section{INTRODUCTION}

Candida albicans is a component of the normal flora of healthy human beings, residing on mucosal surfaces and in the gastrointestinal and genitourinary tracts (Ganguly and Mitchell, 2011). However, when the anatomical barrier is broken or the host immune function is disturbed, C. albicans can cause skin and mucous membrane infection or life-threatening systemic infection (Mayer et al., 2013; Hube et al., 2015). In the United States, C. albicans is the fourth most common microbe causing blood infections with a crude mortality rate of about 50\% (Haynes, 2002; Pfaller and Diekema, 2007). With the increasing severe situation of C. albicans infection, antifungal agents have been developed and widely used in the treatment of various $C$. albicans infections. However, resistance has become a challenge in the clinical treatment of $C$. albicans infections due to wide use or even abuse of antifungal drugs in the recent decade. It is therefore an urgent task to develop new drugs against C. albicans resistant infections (Haque et al., 2016).

The pathogenicity of $C$. albicans depends on various virulence factors, such as adhesion proteins, morphological transformation, secreted aspartyl proteases and phospholipases, phenotypic switching and biofilm formation (Sudbery et al., 2004). There exist at least three morphologies for this organism: a yeast budding form, pseudohyphae, and a filamentous form. C. albicans cells initially adhere to host cells in the yeast budding form, which can grow normally on the mucosal and skin surface of the host without causing immune response. When the growing environment is suitable for hyphal formation, the yeast cells transform to hyphae, which has intensive ability of tissue invasion and infiltration, playing an important role in the pathogenesis (Ramage et al., 2005). Hyphal formation can promote the biofilm formation of C. albicans. Mature biofilms of C. albicans have complex structures consisting of pseudohyphae, hyphae and yeast cells surrounded by exopolymeric matrices, which provide a protective barrier against antifungal therapy, escape cells from the immune system and provide a source of infection for infectious relapse (Bonhomme and d'Enfert, 2013). Thus, inhibiting C. albicans virulence has be recognized as a promising strategy for the treatment of fungal infections as comparing with traditional antifungal drugs targeting cell growth (Biswas et al., 2007).

Natural products have long been an important source of new drug discovery. From 1981 to 2014, nearly 50\% new drugs approved by the United States Food and Drug Administration were either natural product or based thereon (Newman and Cragg, 2012). Kalopanaxsaponin A (KPA) is a triterpenoid saponin isolated from the stem bark of Kalopanax pictus in our laboratory (Figure 1A). Previous studies have showed that KPA has various bioactivities, such as antifungal (Lee et al., 2001), anti-inflammatory (Kim Y.K. et al., 2002; Jeong et al., 2013), antirheumatic (Kim D.H. et al., 2002), anti-tumor (Park et al., 2001) and anti-diabetes (Kim et al., 1998) activities. It has also proved to improve memory deficits by inhibiting the acetylcholinesterase activity (Joh et al., 2012). In this study, we found that KPA could inhibit the proliferation of yeast cells and reduce the virulence of C. albicans by inhibiting adhesion, hyphae and biofilm formation.
Further exploration showed that this inhibitory effect of KPA was attributed to the decrease content of intracellular cyclic adenosine monophosphate (cAMP) and the secretion of farnesol induced by Dpp3 expression. In addition, KPA could prolong the survival time of infected Caenorhabditis elegans in a C. elegansC. albicans infectious model. These finding suggest that KPA may be a promising candidate for use to target fungal virulence against clinically relevant fungal infections.

\section{MATERIALS AND METHODS}

\section{Strains}

Two wild-type strains (SC5314 and YEM30), two azole-resistant strains (CA10 and CA148) (Shandong Province Qianfoshan Hospital, China) (Sun et al., 2009), two mutant strains (DSY448 and DSY1050), and a type culture strain (ATCC10231, Manassas, VA, United States) of C. albicans were used in this study. Wild type C. elegans strain N2 was obtained from the Caenorhabditis Genetics Center, United States. All strains were stored in the medium containing $20 \%$ glycerol at $-80^{\circ} \mathrm{C}$ for a long time. Prior the experiment, C. albicans was inoculated twice on YPD solid plates (yeast extract $1 \%$, peptone $2 \%$, glucose $2 \%$, and agar $2 \%$ ) and cultured at $30^{\circ} \mathrm{C}$. The grown single colonies were inoculated into the liquid YPD broth (2\% tryptone, $1 \%$ yeast extract, and $2 \%$ glucose) and cultured overnight at $30^{\circ} \mathrm{C}, 200 \mathrm{rpm}$.

\section{Chemicals}

Kalopanaxsaponin A was a separated compound extracted from the stem of the Kalopanax in our laboratory, with a purity over $98 \%$ as analyzed by high-performance liquid chromatography. KPA $(10 \mathrm{mg} / \mathrm{mL}$ ) and amphotericin B (AMB, Sigma, $10 \mathrm{mg} / \mathrm{mL}$ ) were dissolved in dimethyl sulfoxide (DMSO) (Sigma, St. Louis, $\mathrm{MO}$, United States) to a stock solution and frozen at $-20^{\circ} \mathrm{C}$ until use. In each assay, the content of DMSO was below $1 \%$.

\section{Minimum Inhibitory Concentration (MIC) Test}

The MIC values of KPA against various genotypes C. albicans were detected by the broth microdilution method as previously described (M27-E4) (Li et al., 2019).

\section{Proliferation Inhibition Test}

The YEM30 strains were diluted to an initial concentration of $1 \times 10^{6}$ cells $/ \mathrm{mL}$ with YPD broth. Add KPA to a final concentration of $0,2,4,8$, and $16 \mu \mathrm{g} / \mathrm{mL}$, and transferred 1 to 96-well flat-bottom plates at $100 \mu \mathrm{L} /$ well. $2 \mu \mathrm{g} / \mathrm{mL}$ of AMB and KPA-free group were served as positive and negative control, respectively. After incubation at $37^{\circ} \mathrm{C}$, the absorbance of $600 \mathrm{~nm}$ was detected every $2 \mathrm{~h}$ with a Bio-Rad Model 680 microplate reader (Bio-Rad Laboratories, Richmond, CA, United States).

\section{Adhesion Assay}

The effect of KPA on C. albicans adhesion was evaluated by the XTT reduction assay as previously described (Li et al., 2019), using AMB $(2 \mu \mathrm{g} / \mathrm{mL})$ as positive control. After culture and wash, 

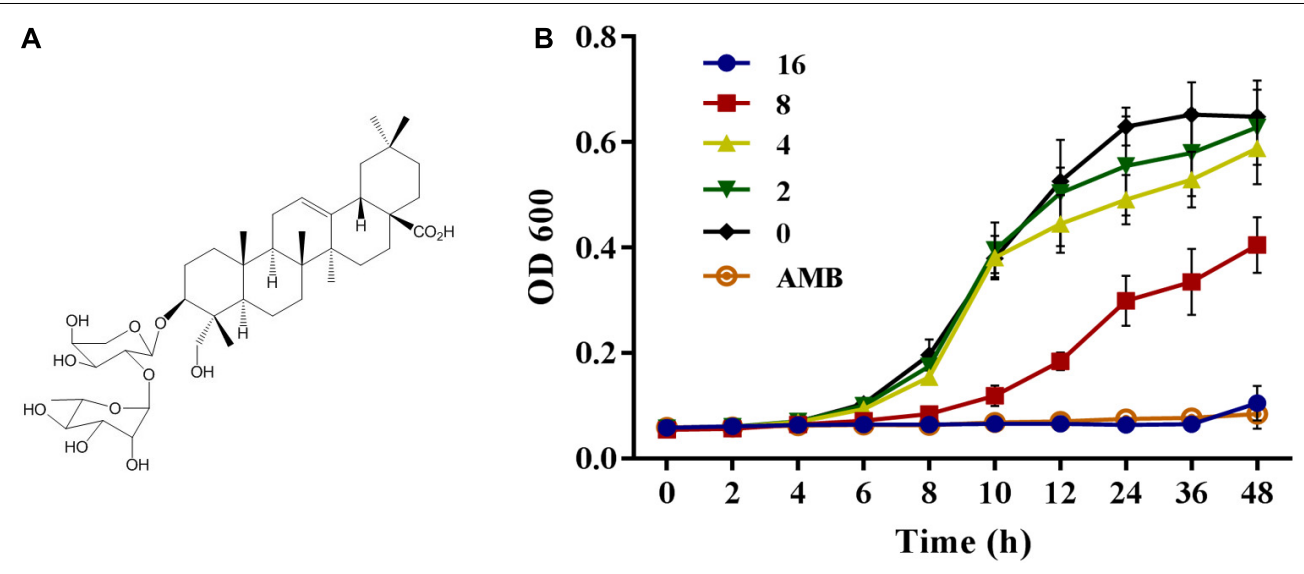

FIGURE 1 | (A) The structure of Kalopanaxsaponin A (KPA). (B) The growth curve of wild type C. albicans strain YEM30 under KPA treatment. YEM30 cells were diluted in the YPD broth and incubated with different doses of KPA at $30^{\circ} \mathrm{C}$. OD600 of each group was detected with a Bio-Rad microplate reader every $1 \mathrm{~h}$. Results were shown as means \pm SDs.

treated YEM30 cells were observed directly under a microscope (Olympus IX71, Olympus, Tokyo, Japan) in the bright field mode. Meanwhile, the remaining adherent cells on the bottom were detected by the XTT reduction assay using an XTT Cell Proliferation Assay Kit (BestBio, Shanghai, China).

\section{Morphological Transition Test}

A YEM30 cell suspension $\left(1 \times 10^{5}\right.$ cells $\left./ \mathrm{mL}\right)$ was prepared in RPMI 1640 containing different concentrations of KPA $(0 \sim 16 \mu \mathrm{g} / \mathrm{mL})$ and incubated at $37^{\circ} \mathrm{C}$ without shaking. The cell morphology was monitored by microscopy (Olympus IX71, Olympus, Tokyo, Japan) every $4 \mathrm{~h}$.

\section{Effect of KPA on C. albicans Biofilm Formation}

The effect of KPA on biofilm formation was detected in 96-well flat-bottom plates using the XTT reduction assay as previously described (Li et al., 2019). YEM30 cells $\left(1 \times 10^{6}\right.$ cells $/ \mathrm{mL}$

TABLE 1 | MIC of KPA and AMB against different genotypes of $C$. albicans in vitro.

\begin{tabular}{lcc}
\hline Strains $^{\dagger}$ & \multicolumn{2}{c}{${\text { MIC of drugs }(\boldsymbol{\mu} \mathbf{g} / \mathbf{m L})^{\ddagger}}^{\ddagger}$} \\
\cline { 2 - 3 } & KPA & AMB \\
\hline ATCC10231 & 16 & 0.5 \\
SC5314 & 16 & 0.5 \\
YEM30 & 16 & 0.5 \\
DSY448 & 16 & 0.5 \\
DSY1050 & 16 & 0.5 \\
CA10 & 16 & 1 \\
CA148 & 16 & 0.5 \\
\hline
\end{tabular}

${ }^{\dagger}$ ATCC10231, SC5314 and YEM30 are wild-type strains. The genotype of DSY448 is $\Delta c$ dr1:hisG-URA3-hisG/Acdr1:hisG; The genotype of DSY1050 is $\quad \Delta c d r 1: h i s G / \Delta c d r 1: h i s G \quad \Delta c d r 2: h i s G / \Delta c d r 2: h i s G \quad \Delta m d r 1: h i s G-U R A 3-h i s G / \Delta$ mdr1:hisG. CA10 and CA148 are clinical isolated azoles multi-resistance C. albicans isolates. ${ }^{\ddagger} K P A$, Kalopanaxsaponin A; AMB, amphotericin $B$. in RPMI 1640) were seeded into a 96-well plate with final concentrations of $0,4,8,16$, and $32 \mu \mathrm{g} / \mathrm{mL}$ of KPA. The plate was incubated for $24 \mathrm{~h}$ at $37^{\circ} \mathrm{C}$ in static culture. The supernatant was removed by three washes with sterile PBS. The XTT reduction assay was performed using XTT Cell Proliferation Assay Kit (BestBio, Shanghai, China), biofilm formation was observed with an Olympus microscope in the bright field mode.

\section{The cAMP Rescue Test}

To determine the effect of cAMP on the filamentation under KPA treatment, YEM30 cells were prepared as for the above morphological transition test. After the addition of $0,12 \mu \mathrm{g} / \mathrm{mL}$ KPA, dibutyryl-cAMP (db-cAMP, Sigma) was added with a final concentration of $1 \mathrm{mM}$. The db-cAMP free groups served as control. The cell morphology was monitored by Olympus microscope every $4 \mathrm{~h}$.

Candida albicans cells $\left(1 \times 10^{5}\right.$ cells $\left./ \mathrm{mL}\right)$ were diluted by RPMI 1640 medium with $16 \mu \mathrm{g} / \mathrm{mL} \mathrm{KPA}$, followed by the addition of db-cAMP at a final concentration of $1 \mathrm{mM}$. The dbcAMP-free cells with or without KPA served as a control. After 24-hour incubation at $37^{\circ} \mathrm{C}$, the medium was discarded, and each well was washed three times with PBS to remove the nonadhered cells. The formed biofilms were imaged microscopically and measured using the XTT reduction assay.

\section{Quantification of Farnesol Secreted in the Supernatant}

YEM30 cells were diluted to $1 \times 10^{5}$ cells/mL with RPMI 1640 medium containing different concentrations of KPA. After 12hour cultured at $37^{\circ} \mathrm{C}, \mathrm{C}$. albicans cells and culture supernatants were collected. Dry weight of $C$. albicans cells was weighted. Farnesol was extracted with the same volume ethyl acetate of supernatants, and the content was quantified using an Agilent model 5975C/7697A GC/MS system as previously described (Li et al., 2015). The content of farnesol was calculated by the standard curve and cell dry weight. 


\section{A Control}

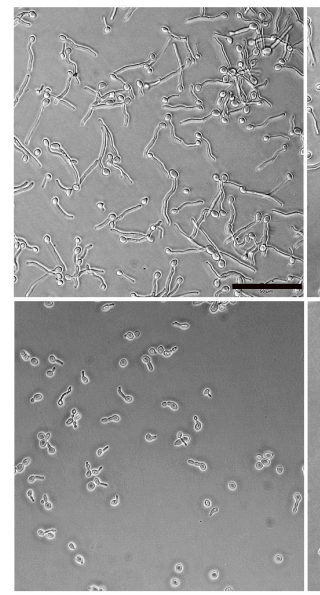

$8 \mu \mathrm{g} / \mathrm{mL}$
$2 \mu \mathrm{g} / \mathrm{mL}$

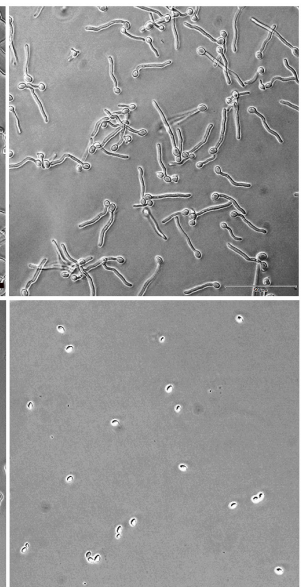

$16 \mu \mathrm{g} / \mathrm{mL}$
$4 \mu \mathrm{g} / \mathrm{mL}$

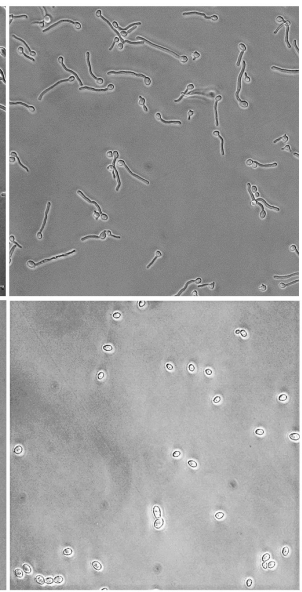

AMB
B

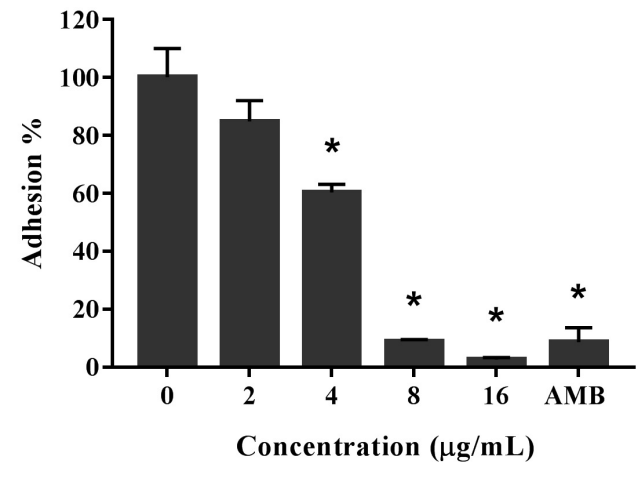

FIGURE 2 | The effect of KPA on the adhesion of $C$. albicans. The effect of TC on the adhesion of $C$. albicans was detected by the microscopy (A) and XTT reduction assay $\mathbf{( B )}$. The bar in panel $\mathbf{( A )}$ indicates $50 \mu \mathrm{m}$. Results in panel $\mathbf{( B )}$ are showed as means \pm SDs. Asterisks $\left(^{*}\right)$ represent significance with $P<0.01$.

\section{Measurement of Dpp3 Expression}

The expression of Dpp3 in BWP17-DPP3-GFP strain was detected after KPA treatment (Zhang et al., 2011). Cells (about $1 \times 10^{5}$ cells $/ \mathrm{mL}$ ) treated by different concentrations of KPA were cultured in RPMI1640 medium at $37^{\circ} \mathrm{C}$ for $3 \mathrm{~h}$. Changes of Dpp3-gfp expression were observed using an Olympus BX53F fluorescence microscope and measured with a BioTek Synergy2 spectrofluorophotometer at $486 \mathrm{~nm}$ excitation and $528 \mathrm{~nm}$ emission wavelengths.

\section{Toxicity Evaluation}

To evaluate the toxicity of KPA, the survival rate of wild type C. elegans was with monitored after KPA treatment. The effect of KPA on the survival of wild type C. elegans N2 was detected as previously described (Chang et al., 2012). Briefly, the prepared nematodes treated with KPA were incubated in a 96-well plate at $25^{\circ} \mathrm{C}$ for 2 days. The survival and death rates were monitored and calculated by counting the numbers of treated worms under the microscope.

\section{C. elegans-C. albicans Infection Model}

C. elegans-C. albicans infectious model was used to evaluate the antifungal activity of KPA in vivo as previously reported (Li et al., 2015). Briefly, worms were exposed to C. albicans strain YEM30 for $2 \mathrm{~h}$ and cultivated with different concentrations of KPA at $25^{\circ} \mathrm{C}$. The survival state of infected worms was monitored daily to calculate survival rates.

\section{Statistical Analysis}

All results were expressed as the mean of the corresponding standard deviation (SD) of three measurements from three independent experiments. The log rank test was used to analyze the data of C. elegans-C. albicans infection assay. The other experimental data were statistically analyzed using Student's $t$-test (two-tailed, unequal variance). $P<0.05$ was considered statistically significant.

\section{RESULTS}

\section{Effect of KPA on Viability of C. albicans Cells}

To evaluate the effect of KPA on C. albicans proliferation, the MIC value of KPA against $C$. albicans of different genotypes

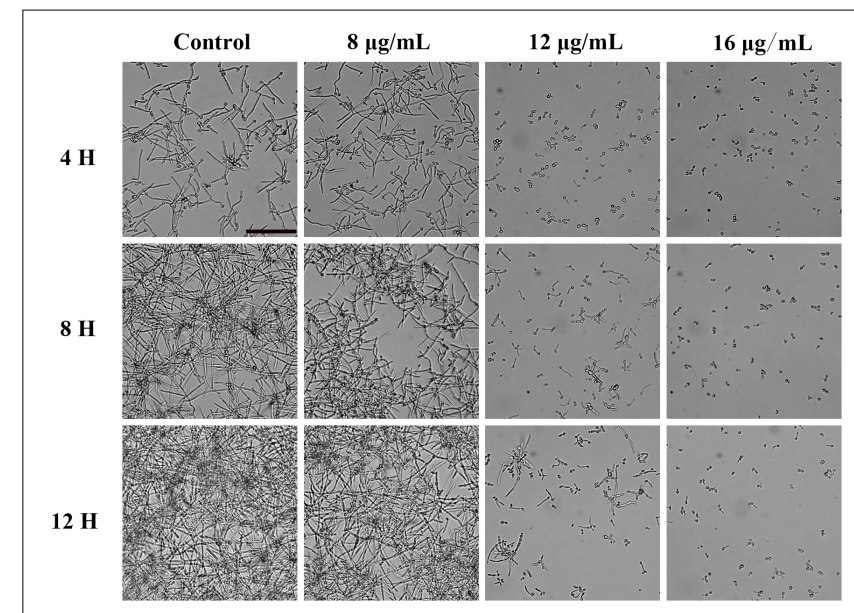

FIGURE 3 | The effect of KPA on the filamentation of C. albicans. YEM30 cells were diluted in the RPMI1640 medium and incubated with different doses of $\mathrm{KPA}$ at $37^{\circ} \mathrm{C}$ without shaking. KPA-free group was served as a negative control. Cells were imaged under a microscope in the bright field every $4 \mathrm{~h}$. The bar indicates $50 \mu \mathrm{m}$. 


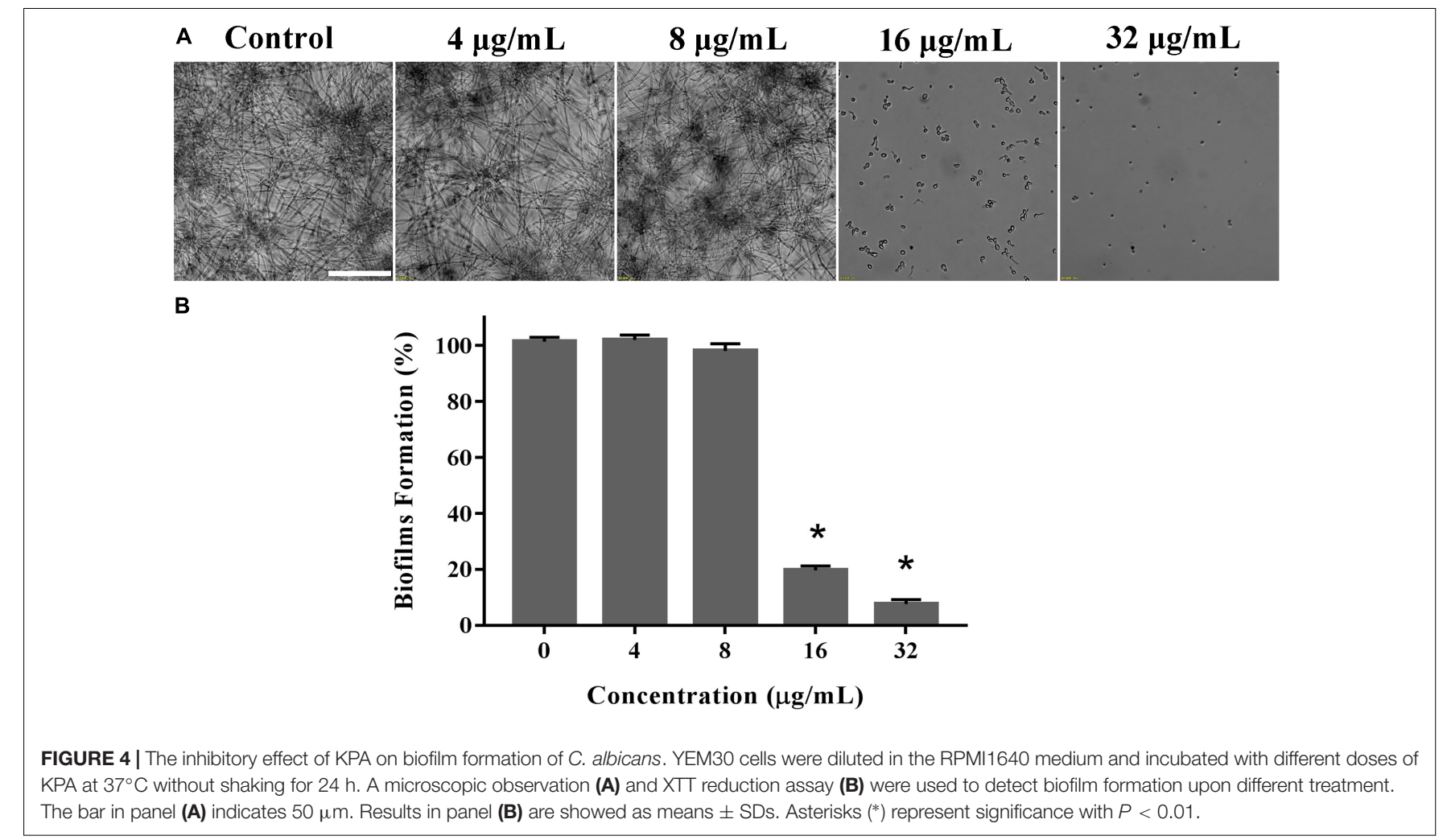

A

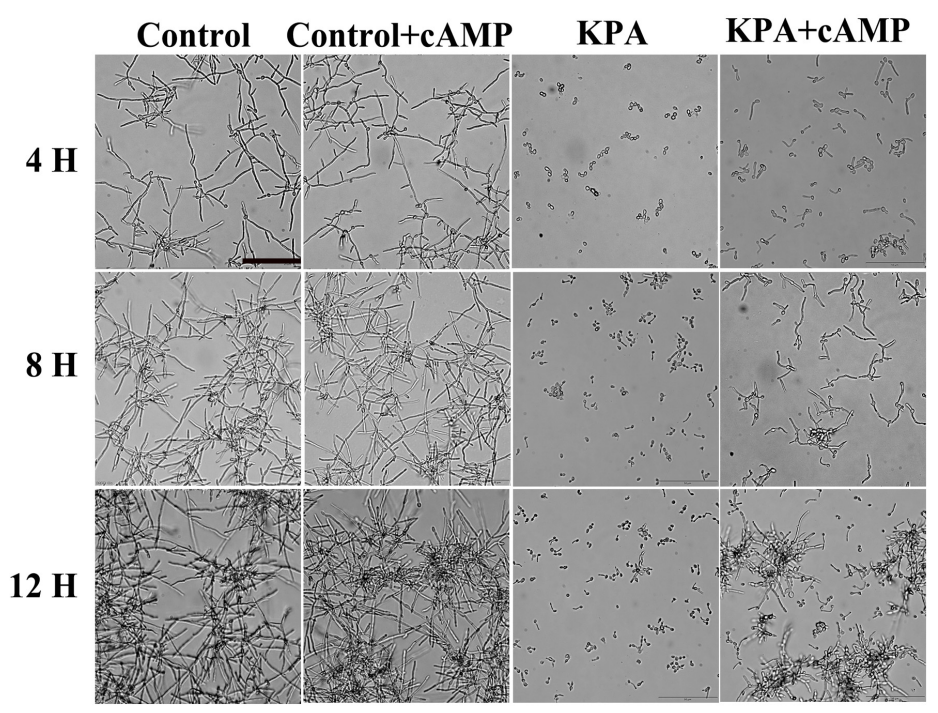

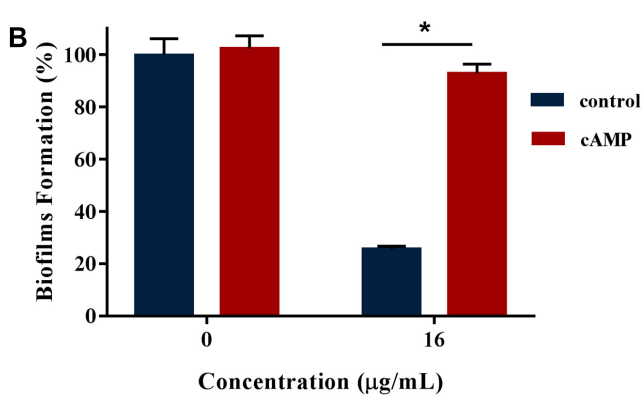

C

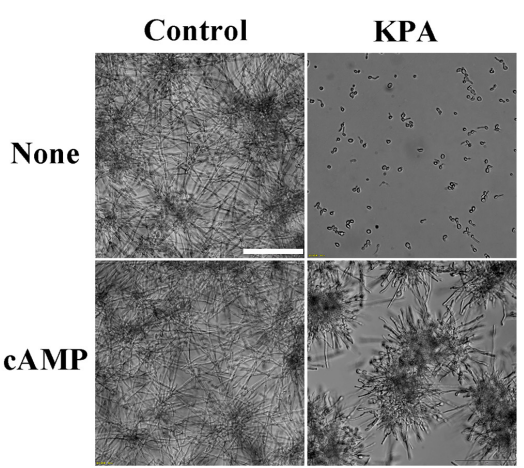

FIGURE 5 | The effect of exogenous cAMP on hyphal and biofilm formation after KPA treatment. (A) Exogenous cAMP restored KPA-inhibited hyphal formation. YEM30 was diluted in RPMl1640 medium with or without $1 \mathrm{mM}$ of db-CAMP and $12 \mu \mathrm{g} / \mathrm{mL}$ of KPA. After 4-hour incubation at $37^{\circ} \mathrm{C}$, cells were imaged under a microscope in the bright field. (B,C) Exogenous CAMP partly restored KPA-inhibited biofilm formation. An XTT reduction assay (B) and microscopic observation (C) were used to detect the formed biofilms upon different treatment. The bars in panels $\mathbf{( A , C )}$ indicate $50 \mu \mathrm{m}$. Results in panel $\mathbf{( B )}$ are showed as means \pm SDs. Asterisks $(*)$ represent significance with $P<0.01$. 
was determined in vitro. It was found that KPA had effective antifungal activity against various genotypes $C$. albicans. As shown in Table 1, the MIC values of all tested strains were $8 \sim 16 \mu \mathrm{g} / \mathrm{mL}(\mathrm{KPA})$ and $0.5 \sim 1 \mu \mathrm{g} / \mathrm{mL}$ (AMB, positive control), respectively.

Besides detection of MIC value, the inhibitory proliferation curve of KPA was also plotted (Figure 1B). Compared with the negative control group, $1 / 2$ MIC $(8 \mu \mathrm{g} / \mathrm{mL})$ of KPA showed an inhibitory effect on the proliferation of C. albicans. The MIC (16 $\mu \mathrm{g} / \mathrm{mL})$ totally inhibited cell growth to a level almost equal to the positive control (AMB, $2 \mu \mathrm{g} / \mathrm{mL}$ ). In conclusion, KPA had significant antifungal activity against $C$. albicans.

\section{Effect of KPA on the Adhesion Ability of C. albicans}

Knowing that the adhesion ability is one of the primary independent factors contributing to the virulence of C. albicans, we detected the effect of KPA on the adhesion of C. albicans at the bottom of the 96-well plates (Sudbery et al., 2004). Microscopically, as shown in Figure 2A, the number of adherent cells under 4,8 , and $16 \mu \mathrm{g} / \mathrm{mL}$ of $\mathrm{KPA}$ was smaller than that of the drug-free group. The same trend was observed in the XTT assay data, showing that 2 and $4 \mu \mathrm{g} / \mathrm{mL} \mathrm{KPA}$ prevented about 10 and $40 \%$ cells from adhering to the substratum of the plate, respectively. The inhibition rate increased to $90 \%$ or nearly $100 \%$ when the concentration was increased to 8 or $16 \mu \mathrm{g} / \mathrm{mL}(P<0.05)$. The inhibition rate in the AMB positive control group was 95\% (Figure 2B).
To sum up, KPA inhibited $C$. albicans adherence in a dosedependent manner.

\section{KPA Inhibited the Yeast-to-Hyphal Transition of $C$. albicans}

Since hyphal development is the main pathogenic factor of C. albicans. We examined the effect of KPA on hyphal formation in vitro by microscopy. Figure 3 showed that KPA effectively inhibited the morphological transformation from yeast to the hyphae. YEM30 cells in 0 and $8 \mu \mathrm{g} / \mathrm{mL}$ groups were able to form regular hyphae within $3 \mathrm{~h}$ and complex networks of yeast within $12 \mathrm{~h} .12 \mu \mathrm{g} / \mathrm{mL}$ of KPA inhibited the occurrence of hyphae within $12 \mathrm{~h}$, while cells of $16 \mu \mathrm{g} / \mathrm{mL}$ KPA kept yeast form. This phenomenon suggested that KPA could inhibit the hyphal formation of C. albicans.

\section{KPA Suppressed the Biofilm Formation of C. albicans}

Biofilms confers intense drug resistance and the immune escape ability C. albicans, indicating that biofilm formation is an important virulence factor of C. albicans (Nett and Andes, 2006). Therefore, we qualitatively analyze the effect of KPA on the film formation microscopically. As shown in Figure 4A, KPA inhibited the biofilm formation completely at the dose of $16 \mu \mathrm{g} / \mathrm{mL}$ or higher, when only a few yeasts were observed at the bottom of the plate. This phenomenon was consistent with the results obtained from the XTT reduction assay (Figure 4B). When the concentration of KPA was increased 16 and $32 \mu \mathrm{g} / \mathrm{mL}$,

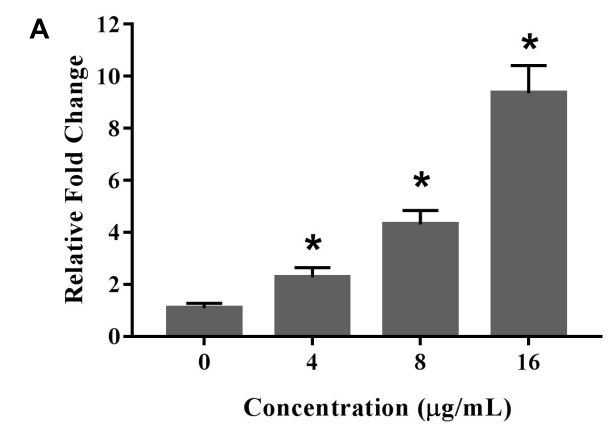

C

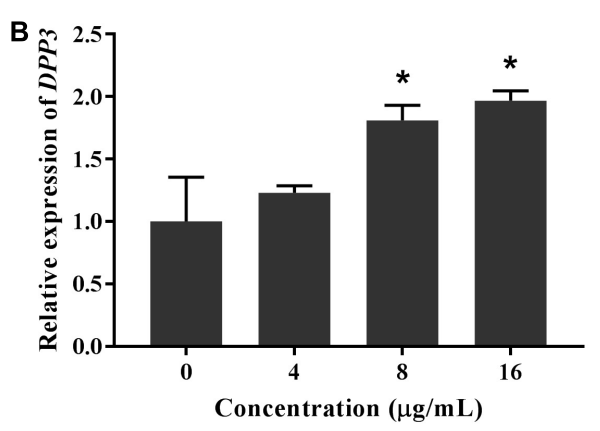

Fluoresence

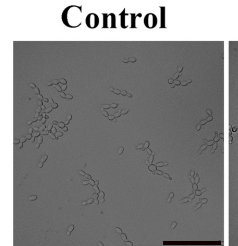

$4 \mu \mathrm{g} / \mathrm{mL}$

$8 \mu \mathrm{g} / \mathrm{mL}$

$16 \mu \mathrm{g} / \mathrm{mL}$
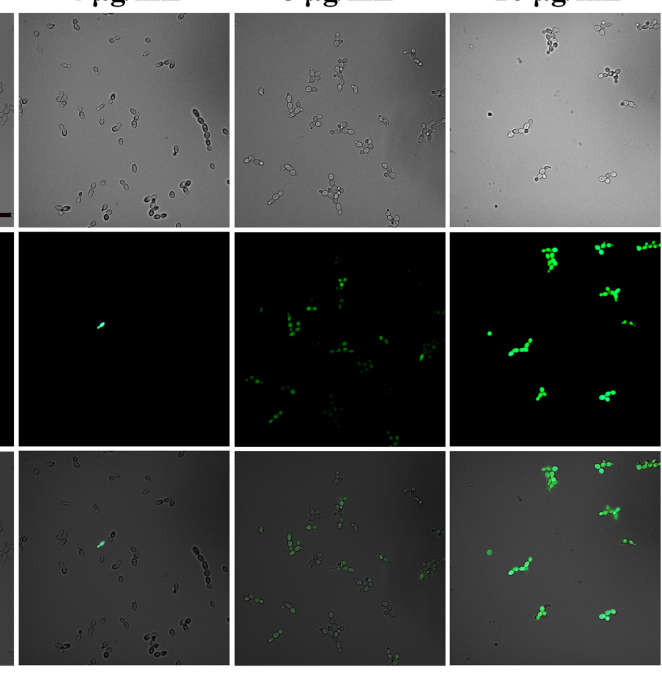

FIGURE 6 | The effect of KPA on farnesol secretion and Dpp3 expression. (A) Farnesol was extracted from KPA cultured supernatants and quantified by GC-MS. $\mathbf{( B , C )}$ The expression of Dpp3 was examined by the fluorescence of BWP17-DPP3-GFP strain. Change in fluorescence was monitored by spectrofluorophotometry (B) and fluorescence microscopy (C). The bar in panel $\mathbf{( A )}$ indicates $50 \mu \mathrm{m}$. Results in panels $\mathbf{( A , B )}$ are showed as means \pm SDs. Asterisks (*) in panels (A,B) represent significance with $P<0.05$. 
the inhibition rate of biofilms was 80 and $90 \%$, respectively, suggesting that KPA could suppress biofilm formation by inhibiting the yeast-to-hyphal transition of C. albicans.

\section{cAMP Rescues the Inhibition Effect of KPA on Hyphae and Biofilm Formation}

Cyclic adenosine monophosphate is the core molecule of regulatory network of morphological transformation, which can positively regulate the hyphal formation process of yeast cells (Sudbery, 2011). We conducted cAMP rescue experiment to observe whether cAMP affected the inhibitory effect of KPA on hyphae and biofilm formation. The results showed that the hyphae and biofilm growth inhibited by KPA $(16 \mu \mathrm{g} / \mathrm{mL})$ was restored by the addition of $1 \mathrm{mM}$ exogenous db-cAMP within 12 and $24 \mathrm{~h}$ incubation (Figures 5A,C). The addition of db-cAMP restored the growth rate of yeast biofilms from 25.58 to $92.91 \%$ (Figure 5B). The restored effect of cAMP was continuous rather than transient. These results revealed that KPA could inhibit the growth of hyphae and biofilms by reducing the content of cAMP in yeast cells.

\section{KPA Stimulates Farnesol Secretion by Regulating Dpp3 Expression}

Farnesol, known as a quorum sensing molecule secreted by C. albicans, can inhibit the transition from yeast to hyphae. Therefore, we examined the effect of KPA on farnesol secretion. The result of GC-MS test results showed that KPA had a positive effect on the farnesol content of the supernatant. Compared with the control group, 4, 8, and $16 \mu \mathrm{g} / \mathrm{mL}$ of KPA treatment could increase the secretion of farnesol by $2.39,4.16$, and 9.15 times (Figure 6A).

Dpp3 is the key enzyme in the synthesis of farnesol (Navarathna et al., 2007). A Dpp3 labeled green fluorescent protein strain (BWP17-DPP3-GFP) was used for detecting the effect of KPA on Dpp3 expression. The results showed that the fluorescence intensity of Dpp3-gfp was positively correlated with the dose of KPA (Figure 6C). Similar to farnesol, KPA increased the DPP3 expression in a dose-dependent manner (Figure 6B). From the above results, we concluded that KPA could induce the synthesis of farnesol by stimulating the expression of DPP3, eventually inhibiting hyphal formation.

\section{KPA Prolonged the Survival Time of C. albicans Infected C. elegans}

Given the antifungal activity of KPA in vitro, we used the C. elegans-C. albicans infectious model to further investigate the antifungal activity of KPA in vivo. The survival curve showed that the nematode survival time of KPA treated group $(8,16 \mu \mathrm{g} / \mathrm{mL})$ in the infectious model was longer than the control group (Figure 7B). In addition, the result of toxicity test indicated that high doses of KPA (4-fold of MIC) did not affect the survival of healthy wild type C. elegans within 2 days (Figure 7A). These data demonstrated the potential value of KPA for the treatment on C. albicans infection in vivo.

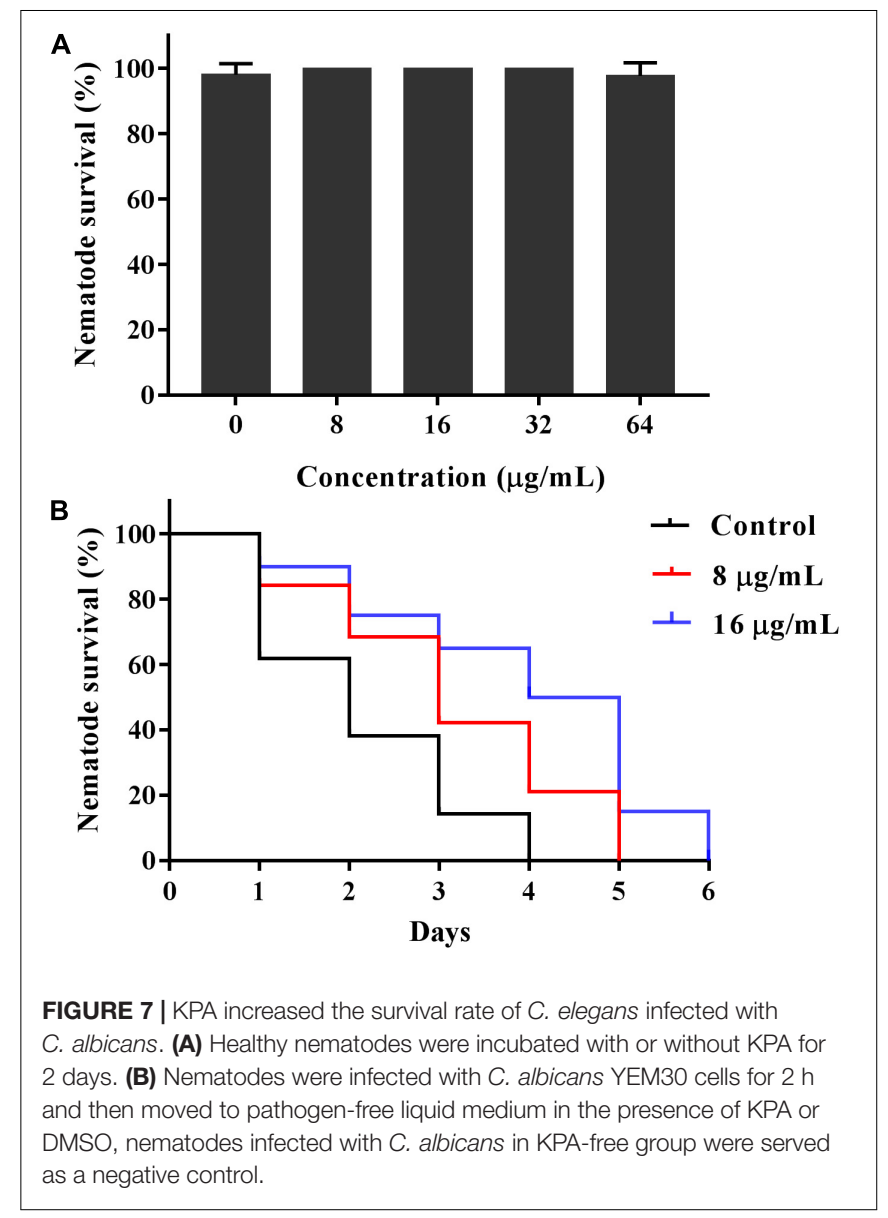

\section{DISCUSSION}

As an opportunistic pathogen, C. albicans can survive at several anatomically different sites, adapt to environment changes through morphological transformation and gradually form biofilms, causing fatal infections in immunodeficient patients (Noble et al., 2010). Morphological transformation is known as the most critical virulence factor inducing the biofilm formation in resistance to a variety of antifungal drugs including amphotericin B, acanthomycin and azole (Gulati and Nobile, 2016). The increasing emergence of drug resistance has increased the demand for new antifungal drugs and new antifungal strategies. It was found in this study that KPA had antifungal activity against $C$. albicans, and $1 / 2 \mathrm{MIC}$ effectively blocked the growth of yeast cells, indicating that low dose antifungal activity and high dose fungicidal activity of KPA. The results of MIC test showed that antifungal effect of KPA was independent of efflux regulatory proteins, including the ATP-binding cassette (ABC) transporter family members $\mathrm{Cdr} 1$ and $\mathrm{Cdr2}$, and the major facilitator Mdr1. Efflux pump gene overexpression of is a vital reason for drug resistance (Cowen et al., 2015), suggesting KPA had a low probability to form resistance. In addition, the C. elegans-C. albicans infectious model showed that KPA protected $C$. elegans against infection through inhibiting yeast 
cells proliferation. More importantly, the low toxicity of KPA to healthy C. elegans increased the value of further research on KPA.

Hyphal formation is an amusing feature of C. albicans, which plays an important role in adhesion and biofilm formation (Modrzewska and Kurnatowski, 2015; Carradori et al., 2016). Given this characteristic, invasive hyphae are often detected in tissues of infected hosts. Correspondingly, hyphal defective C. albicans often exhibits lower virulence (Jabra-Rizk et al., 2016). Arresting or preventing morphogenesis means stopping infection. It was found in this study that KPA could reduce the virulence of $C$. albicans by inhibiting adhesion, yeast-to-hyphal transition and biofilm formation. These findings gave us further interest to explore its molecular mechanism.

Hyphal formation is influenced by various environmental factors such as quorum-sensing molecules (Sudbery, 2011). Farnesol, as a precursor of the isoprene/sterol pathway, is a quorum sensing molecule produced by microorganisms which is involved in preventing the morphological transformation and biofilm development of C. albicans, with cytotoxicity at a certain concentration (Shirtliff et al., 2009). DPP3 is a gene encoding synthase of farnesol, and its expression was reported to affect the secretion of farnesol (Navarathna et al., 2007). The results demonstrated that KPA increased the secretion of farnesol by up-regulating Dpp3 expression. The development of hyphae is a complex process. Environmental factors are only external inducements of the morphological transformation, and regulation of the signal transduction pathway is believed to be the real internal cause (Sudbery, 2011). cAMP, synthesized by Cdc35, is considered as a key molecule in Ras1-cAMP-Efg1 pathway. It is a strong regulator for hyphal formation. Previous study has been proved that inhibition of cAMP synthesis could block yeast form growth of $C$. albicans under most hyphal induction conditions (Zhang et al., 2017). Furthermore, farnesol could regulate Ras-cAMP-Efgl pathway by inhibiting the activity of Cdc35 to inhibit the development of hyphae (Lindsay et al., 2016). Ulterior exploration indicated that exogenous cAMP could restore the growth of hyphae and biofilms inhibited by KPA. The decrease of intracellular

\section{REFERENCES}

Biswas, S., Van Dijck, P., and Datta, A. (2007). Environmental sensing and signal transduction pathways regulating morphopathogenic determinants of Candida albicans. Microbiol. Mol. Biol. Rev. 71, 348-376. doi: 10.1128/MMBR.00009-06

Bonhomme, J., and d'Enfert, C. (2013). Candida albicans biofilms: building a heterogeneous, drug-tolerant environment. Curr. Opin. Microbiol. 16, 398-403. doi: 10.1016/j.mib.2013.03.007

Carradori, S., Chimenti, P., Fazzari, M., Granese, A., and Angiolella, L. (2016). Antimicrobial activity, synergism and inhibition of germ tube formation by crocus sativus-derived compounds against Candida spp. J. Enzyme Inhib. Med. Chem. 31(Suppl. 2), 189-193. doi: 10.1080/14756366.2016.1180596

Chang, W., Li, Y., Zhang, L., Cheng, A., and Lou, H. (2012). Retigeric acid B attenuates the virulence of Candida albicans via inhibiting adenylyl cyclase activity targeted by enhanced farnesol production. PLoS One 7:e41624. doi: 10.1371/journal.pone.0041624

Cowen, L. E., Sanglard, D., Howard, S. J., Rogers, P. D., and Perlin, D. S. (2015). Mechanisms of antifungal drug resistance. Cold Spring Harb. Perspect. Med. 5:a019752. doi: 10.1101/cshperspect.a019752
cAMP induced by KPA could directly inhibit filamentation of C. albicans.

In this study, we found that KPA could inhibit proliferation and various virulence factors of $C$. albicans, suggesting that KPA might be a promising antifungal agent to prevent Candida infection due to its low toxicity and resistance. Nevertheless, in vivo studies need to also be carried out to determine its biocompatibility, cytotoxicity, safety, and mode of action of KPA before it further used for biomedical applications.

\section{DATA AVAILABILITY STATEMENT}

All datasets generated for this study are included in the article/supplementary material.

\section{AUTHOR CONTRIBUTIONS}

HY participated in the extraction of KPA. HL and YL conceived the study and designed the experimental procedures. YL, MS, YW, and MY performed the experiments. YL, MS, HY, ZZ, and BG analyzed the data. ZZ, YL, and MS wrote the manuscript.

\section{FUNDING}

This research was supported by the National Natural Science Foundation of China (81701390 and 81902040), Natural Science Foundation of Jiangsu Province (BK20170250), Postdoctoral Science Foundation of Jiangsu Province (2018K245C), and Xuzhou Science and Technology Innovation Project (KC19057).

\section{ACKNOWLEDGMENTS}

Part of the experiment in this study was completed in the Public Experimental Research Center of Xuzhou Medical University and thanks the teachers for their support and help during the experiments.

Ganguly, S., and Mitchell, A. P. (2011). Mucosal biofilms of Candida albicans. Curr. Opin. Microbiol. 14, 380-385. doi: 10.1016/j.mib.2011.06.001

Gulati, M., and Nobile, C. J. (2016). Candida albicans biofilms: development, regulation, and molecular mechanisms. Microbes Infect. 18, 310-321. doi: 10. 1016/j.micinf.2016.01.002

Haque, F., Alfatah, M., Ganesan, K., and Bhattacharyya, M. S. (2016). Inhibitory effect of sophorolipid on Candida albicans biofilm formation and hyphal growth. Sci. Rep. 6:23575. doi: 10.1038/srep23575

Haynes, K. (2002). Virulence in Candida species. Trends Microbiol. 9, 591-596. doi: 10.1016/S0966-842X(01)02237-5

Hube, B., Hay, R., Brasch, J., and Veraldi, S. (2015). Schaller M. Dermatomycoses and inflammation: the adaptive balance between growth, damage, and survival. J. Mycol. Med. 25, e44-e58. doi: 10.1016/j.mycmed.2014.11.002

Jabra-Rizk, M. A., Kong, E. F., Tsui, C., Nguyen, M. H., Clancy, C. J., Fidel, P. L. Jr., et al. (2016). Candida albicans pathogenesis: fitting within the host-microbe damage response framework. Infect. Immun. 84, 2724-2739. doi: 10.1128/IAI. 00469-16

Jeong, Y. H., Hyun, J. W., Kim Van Le, T., Kim, D. H., and Kim, H. S. (2013). Kalopanaxsaponin A exerts anti-inflammatory effects in 
lipopolysaccharide-stimulated microglia via inhibition of JNK and NFКВ/AP-1 pathways. Biomol. Ther. 21, 332-337. doi: 10.4062/biomolther. 2013.069

Joh, E. H., Lee, I. A., and Kim, D. H. (2012). Kalopanaxsaponins A and B Isolated from Kalopanax pictus ameliorate memory deficits in mice. Phytother. Res. 26, 546-551. doi: 10.1002/ptr.3596

Kim, D. H., Yu, K. W., Bae, E. A., Park, H. J., and Choi, J. W. (1998). Metabolism of Kalopanaxsaponin B and $\mathrm{H}$ by human intestinal bacteria and antidiabetic activity of their metabolites. Biol. Pharm. Bull. 21, 360-365. doi: 10.1248/bpb. 21.360

Kim, Y. K., Kim, R. G., Park, S. J., Ha, J. H., Choi, J. W., Park, H. J., et al. (2002). In vitro antiinflammatory activity of Kalopanaxsaponin A isolated from kalopanax pictus in murine macrophage RAW 264.7 cells. Biol. Pharm. Bull. 25, 472-476. doi: 10.1248/bpb.25.472

Kim, D. H., Bae, E. A., Han, M. J., Park, H. J., and Choi, J. W. (2002). Metabolism of Kalopanaxsaponin $\mathrm{K}$ by human intestinal bacteria and antirheumatoid arthritis activity of their metabolites. Biol. Pharm. Bull. 25, 68-71. doi: 10.1248/bpb. 25.68

Lee, M. W., Kim, S. U., and Hahn, D. R. (2001). Antifungal activity of modified hederagenin glycosides from the leaves of Kalopanax pictum var. chinense. Biol. Pharm. Bull. 24, 718-719. doi: 10.1248/bpb.24.718

Li, Y., Chang, W., Zhang, M., Ying, Z., and Lou, H. (2015). Natural product solasodine-3-O- $\beta$-D-glucopyranoside inhibits the virulence factors of Candida albicans. FEMS Yeast Res. 15:fov060. doi: 10.1093/femsyr/fov060

Li, Y., Shan, M., Yan, M., Xu, Y., Wang, Y., Chen, Y., et al. (2019). The inhibition of trans-cinnamaldehyde on the virulence of Candida albicans via enhancing farnesol secretion with low potential for the development of resistance. Biochem. Biophys. Res. Commun. 515, 544-550. doi: 10.1016/j.bbrc.2019.05.165

Lindsay, A. K., Deveau, A., Piispanen, A. E., and Hogan, D. A. (2016). Farnesol and cyclic AMP signaling effects on the hypha-to-yeast transition in Candida albicans. Eukaryot. Cell 11, 1219-1225. doi: 10.1128/EC.00144-12

Mayer, F. L., Wilson, D., and Hube, B. (2013). Candida albicans pathogenicity mechanisms. Virulence 4, 119-128. doi: 10.4161/viru.22913

Modrzewska, B., and Kurnatowski, P. (2015). Adherence of Candida sp. to host tissues and cells as one of its pathogenicity features. Ann. Parasitol. 61, 3-9.

Navarathna, D. H., Hornby, J. M., Krishnan, N., Parkhurst, A., Duhamel, G. E., and Nickerson, K. W. (2007). Effect of farnesol on a mouse model of systemic candidiasis, determined by use of a DPP3 knockout mutant of Candida albicans. Infect. Immun. 75, 1609-1618. doi: 10.1128/IAI.01182-06

Nett, J., and Andes, D. (2006). Candida albicans biofilm development, modeling a host-pathogen interaction. Curr. Opin. Microbiol. 9, 340-345. doi: 10.1016/j. mib.2006.06.007

Newman, D. J., and Cragg, G. M. (2012). Natural products as sources of new drugs over the 30 years from 1981 to 2010. J. Nat. Prod. 75, 311-335. doi: $10.1021 / \mathrm{np} 200906 \mathrm{~s}$
Noble, S. M., French, S., Kohn, L. A., Chen, V., and Johnson, A. D. (2010). Systematic screens of a Candida albicans homozygous deletion library decouple morphogenetic switching and pathogenicity. Nat. Genet. 42, 590-598. doi: 10. 1038/ng.605

Park, H. J., Kwon, S. H., Lee, J. H., Lee, K. H., Miyamoto, K., and Lee, K. T. (2001). Kalopanaxsaponin A is a basic saponin structure for the anti-tumor activity of hederagenin monodesmosides. Planta Med. 67, 118-121. doi: 10.1055/s-200111516

Pfaller, M. A., and Diekema, D. J. (2007). Epidemiology of invasive candidiasis: a persistent public health problem. Clin. Microbiol. Rev. 20, 133-163. doi: 10. 1128/cmr.00029-06

Ramage, G., Saville, S. P., Thomas, D. P., and López-Ribot, J. L. (2005). Candida biofilms: an update. Eukaryot. Cell 4, 633-638. doi: 10.1128/EC.4.4.633-638. 2005

Shirtliff, M. E., Krom, B. P., Meijering, R. A., Peters, B. M., Zhu, J., Scheper, M. A., et al. (2009). Farnesol-induced apoptosis in Candida albicans. Antimicrob. Agents Chemother. 53, 2392-2401. doi: 10.1128/AAC.01551-08

Sudbery, P., Gow, N., and Berman, J. (2004). The distinct morphogenic states of Candida albicans. Trends Microbiol. 12, 317-324. doi: 10.1016/j.tim.2004. 05.008

Sudbery, P. E. (2011). Growth of Candida albicans hyphae. Nat. Rev. Microbiol. 9, 737-748. doi: 10.1038/nrmicro2636

Sun, L., Sun, S., Cheng, A., Wu, X., Zhang, Y., and Lou, H. (2009). In vitro activities of retigeric acid $\mathrm{B}$ alone and in combination with azole antifungal agents against Candida albicans. Antimicrob. Agents Chemother. 53, 1586-1591. doi: 10.1128/AAC.00940-08

Zhang, L., Chang, W., Sun, B., Groh, M., Speicher, A., and Lou, H. (2011). Bisbibenzyls, a new type of antifungal agent, inhibit morphogenesis switch and biofilm formation through upregulation of DPP3 in Candida albicans. PLoS One 6:e28953. doi: 10.1371/journal.pone.0028953

Zhang, M., Chang, W., Shi, H., Zhou, Y., Zheng, S., Li, Y., et al. (2017). Biatriosporin D displays anti-virulence activity through decreasing the intracellular cAMP levels. Toxicol. Appl. Pharmacol. 322, 104-112. doi: 10.1016/ j.taap.2017.03.004

Conflict of Interest: The authors declare that the research was conducted in the absence of any commercial or financial relationships that could be construed as a potential conflict of interest.

Copyright (c) $2019 \mathrm{Li}$, Shan, Yan, Yao, Wang, Gu, Zhu and Li. This is an open-access article distributed under the terms of the Creative Commons Attribution License (CC BY). The use, distribution or reproduction in other forums is permitted, provided the original author(s) and the copyright owner(s) are credited and that the original publication in this journal is cited, in accordance with accepted academic practice. No use, distribution or reproduction is permitted which does not comply with these terms. 